\title{
COVID-19: a historicidade das terapêuticas no primeiro ano da Pandemia
}

\author{
COVID-19: the historicity of therapies in the first year of the Pandemic \\ COVID-19: la historicidad de las terapias en el primer año de la Pandemia
}

Recebido: 29/01/2022 | Revisado: 02/02/2022 | Aceito: 04/02/2022 | Publicado: 06/02/2022

\author{
Daniel Andolfatto \\ ORCID: https://orcid.org/0000-0003-4872-5361 \\ Universidade Comunitária da Região de Chapecó, Brasil \\ E-mail: daniel.andolfatto@unochapeco.edu.br \\ Leila Zanatta \\ ORCID: https://orcid.org/0000-0003-0935-4190 \\ Universidade Estadual de Santa Catarina, Brasil \\ E-mail: leila.zanatta@gmail.com.br \\ Lucimare Ferraz \\ ORCID: https://orcid.org/0000-0002-2487-8614 \\ Universidade Comunitária da Região de Chapecó, Brasil \\ E-mail: lferraz@unochapeco.edu.br
}

\begin{abstract}
Resumo
Objetiva-se conhecer os construtos terapêuticos da COVID-19 em seu primeiro ano de pandemia. Trata-se de uma revisão narrativa da literatura, realizada a partir de pesquisas nas bases Biblioteca Virtual de Saúde, Periódicos Capes, PubMed, Cochrane com os descritores padronizados 'Therapy' AND COVID-19, no primeiro ano da pandemia da COVID-19, após a declaração oficial da OMS. Como resultados foram descritos os alvos terapêuticos pesquisados (in vivo, in vitro e in silico) e publicados no primeiro ano dessa crise sanitária. Evidenciou-se que as terapias exploradas se basearam em alvos farmacológicos e não farmacológicos sobre o hospedeiro, sistema imunológico e no vírus. Os principais agentes testados in vitro e in vivo e in silico pertences as classes farmacológicas dos antivirais, antiparasitários, anti-inflamatórios, antibióticos além de anticorpos monoclonais, células-tronco e plasma convalescente. Segundo as publicações, os medicamentos mais citados para tratar a COVID-19 foram a hidroxicloroquina, cloroquina, ivermectina, azitromicina, vitamina $\mathrm{D}$, dexametasona ou outro glicocorticoide. Constatou-se que muitas estratégias terapêuticas contra a COVID-19 foram refutadas, todavia outras estão sendo melhor pesquisadas.
\end{abstract}

Palavras-chave: SARS-CoV-2; Terapias; Pandemia; COVID-19.

\begin{abstract}
The objective is to know the therapeutic constructs of COVID-19 in its first year of the pandemic. This is a narrative review of the literature, carried out from research in the Biblioteca Virtual de Saúde, Periódicos Capes, PubMed, Cochrane with the standardized descriptors 'Therapy' AND COVID-19, in the first year of the COVID-19 pandemic, after the official declaration of the WHO. As a result, the researched therapeutic targets (in vivo, in vitro, in silico) and published in the first year of this health crisis were described. It was evident that the therapies explored were based on pharmacological and non-pharmacological targets on the host, immune system the virus. The main agents tested in vitro, in vivo, in silico belong to the pharmacological classes of antivirals, antiparasitics, anti-inflammatories, antibiotics as well as monoclonal antibodies, stem cells convalescent plasma. According to the publications, the most cited drugs to treat COVID-19 were hydroxychloroquine, chloroquine, ivermectin, azithromycin, vitamin D, dexamethasone glucocorticoid. It was found that many therapeutic strategies against COVID-19 have been refuted, however, others are being better researched.
\end{abstract}

Keywords: SARS-CoV-2; Therapies; Pandemic; COVID-19.

\section{Resumen}

El objetivo es conocer los constructos terapéuticos de la COVID-19 en su primer año de pandemia. Esta es una revisión narrativa de la literatura, realizada a partir de investigaciones en la Biblioteca Virtual de Saúde, Periódicos Capes, PubMed, Cochrane con los descriptores estandarizados 'Terapia' Y COVID-19, en el primer año de la pandemia de COVID-19, después la declaración oficial de la OMS. Como resultado se describieron las dianas terapéuticas investigadas (in vivo, in vitro e in silico) y publicadas en el primer año de esta crisis sanitaria. Era evidente que las terapias exploradas se basaban en dianas farmacológicas y no farmacológicas sobre el huésped, el sistema inmunitario y el virus. Los principales agentes probados in vitro e in vivo e in silico pertenecen a las clases farmacológicas de antivirales, antiparasitarios, antiinflamatorios, antibióticos, así como anticuerpos monoclonales, células madre y plasma convaleciente. Según las publicaciones, los fármacos más citados para tratar la COVID-19 fueron la hidroxicloroquina, la cloroquina, la ivermectina, la azitromicina, la vitamina $\mathrm{D}$, la dexametasona u otro 
glucocorticoide. Se encontró que muchas estrategias terapéuticas contra el COVID-19 han sido refutadas, sin embargo, otras están siendo mejor investigadas.

Palabras clave: SARS-CoV-2; Terapias; Pandemia; COVID-19.

\section{Introdução}

Numa linha cronológica, em 8 de dezembro de 2019 começaram a ser relatados casos de pneumonia com etiologia desconhecida na cidade de Wuhan, China, (Chen et al., 2020) e no dia 31 de dezembro de 2019, a sede da Organização Mundial da Saúde (OMS) no país foi notificada acerca desses casos emergentes, assim, em 7 de janeiro de 2020 o vírus SARSCoV-2 foi isolado e identificado a partir de uma amostra de esfregaço da garganta (UNA-SUS, 2020). A partir disso, em 9 de janeiro de 2020 foi confirmada a sua rápida circulação. Em 16 de janeiro já houve a notificação do primeiro caso em território japonês e em 21 de janeiro o primeiro caso nos Estados Unidos da América (Lana et al., 2020). Já em 23 de janeiro de 2020, havia 830 casos confirmados na China e 09 casos relatados em outros países e em menos de 60 dias, no dia 29 de janeiro de 2020, o vírus havia se espalhado para 29 províncias e regiões da China, contabilizando 1287 casos confirmados,1965 casos suspeitos e 41 mortes (Q. Li et al., 2020). Com isso, em 30 de janeiro de 2020 a OMS declarou o surto de Coronavírus como Emergência de Saúde Pública de Importância Internacional e em 11 de março declarou estado de pandemia, contando com 110 mil casos e 4 mil óbitos espalhados por todos os continentes (Garcia \& Duarte, 2020).

Exato um ano após a declaração da pandemia pela OMS, marco histórico da crise da saúde no mundo moderno, às 17h04 do dia 11 de março de 2021 registrou-se globalmente 117.799.584 de casos confirmados, incluindo 2.615.018 mortes notificadas à OMS. E em 10 de março de 2021, definiu-se como principal medida de combate à pandemia a administração de uma vacina; assim, foram administrados um total de 300.002.228 doses (OMS, 2021).

O SARS-CoV-2 trata-se de vírus zoonótico da família do coronavírus que provoca a Síndrome do Desconforto Respiratória Aguda. Além de problemas orgânicos-patológicos, a pandemia da COVID-19 desencadeou diferentes problemas sociais e sanitários, tornando-se uma crise mundial, transcendendo a saúde, e atingindo os aspectos antropocênicos, naturais, sociais e geopolítico. Pois, mesmo realizando as orientações preconizadas, qualquer pandemia conturba as relações sociais, culturais e econômicas do mundo inteiro. E o mais grave "assolando" famílias, educação e a saúde das pessoas (Bender et al., 2014).

Contudo, ao longo deste um ano de pandemia não foram suficientes todos os esforços para encontrar terapias eficazes e seguras, bastando assim, reunir esforços com projetos internacionais como Solidarity, conduzido por uma comissão da Organização Mundial de Saúde para comprovar cientificamente uma terapia eficaz para a COVID-19 (OMS, 2021).

$\mathrm{Na}$ medida em que vislumbra impactos sociais negativos, sofridos por um problema sanitário, como a COVID-19, Cueto (2020) destaca a importância da pesquisa histórica para compreender o passado e o presente, pois são esses estudos que embasam outros conhecimentos, tecendo novas reorganizações e planejamentos pragmáticos, objetivando a redução dos problemas sociais.

Nesse cenário de crise sanitária e social, torna-se importante aglutinar e resumir as informações acerca das possibilidades terapêuticas que foram utilizadas e publicadas no meio científico. Assim, este estudo objetivou conhecer os construtos terapêuticos da COVID-19 referente às estratégias farmacológicas e não farmacológicas, segundo as publicações científicas no primeiro ano da maior pandemia da história contemporânea.

\section{Metodologia}

Para o desenvolvimento dessa pesquisa, optou-se por um estudo narrativo dos manuscritos publicizados, entre 11 de março de 2020 à 10 de março de 2021, nas fontes de dados Cochrane, Periódicos Capes, PubMed e Biblioteca Virtual de 
Saúde. Para o autor Mota de Souza et al. (2018) os estudos de revisão narrativa, são publicações amplas, em que os materiais utilizados são analisados de forma generalizada, afim de responder os objetivos propostos.

A importância de utilizar uma revisão narrativa para a problemática, deve-se à situação de emergência de saúde internacional, a COVID-19, por ser uma doença nova/emergente em que todo material publicizado e validado sobre ela, nesse momento é relevante. Desde modo, outros tipos de revisões não seriam capazes de considerar todos os aspectos e fontes de informações que contribuem para compressão da Pandemia. Portanto, essa revisão narrativa, pretende construir o 'estado de arte' da terapêutica da COVID-19 em seu primeiro ano de Pandemia, evidenciando as diversas fontes de informações, sejam elas de materiais publicados em bases de dados de periódicos científicos, como também de instituições governamentais e balizadoras de práticas de saúde, como OMS e OPAS.

Para compor essa revisão, a busca das publicações ocorreu com os descritores/termos na linguagem inglesa: Therapy AND COVID-19. Contudo, os seguintes critérios de inclusão foram considerados: materiais, em diferentes idiomas, publicizados e revisados por pares: artigos, relato de casos, editoriais. Também serão incluídos documentários, notas técnicas, boletins epidemiológicos, reportagens, documentário, entre outros.

Por meio dessa estratégia de busca, identificou-se 394 artigos. Após considerar os critérios de exclusão e inclusão, foram selecionados 111 para compor essa narrativa conforme a Figura 1.

Figura 1 - Fluxograma/Prisma de identificação e seleção dos artigos (2021).

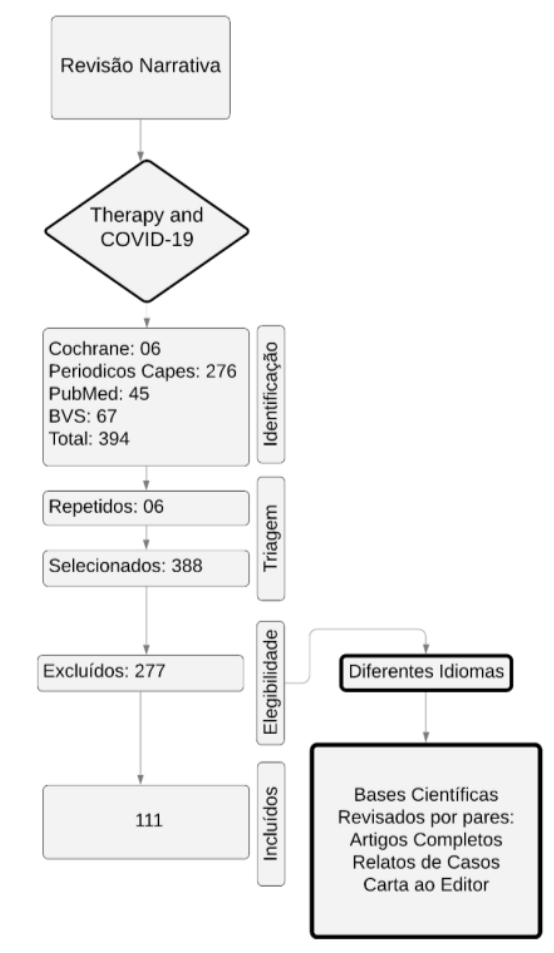

Fonte: Elaborado pelos autores (2021).

\section{Resultados e Discussão}

No fim de março de 2020, a OMS esclareceu sobre as terapias que estavam sendo utilizadas para tratar a COVID-19, alertando que não havia naquele momento nenhum produto farmacêutico que se mostrou seguro e eficaz.

No início de julho de 2020 a OMS realizou a segunda cúpula sobre pesquisa e inovação para a COVID-19, com o objetivo de realizar um balanço da ciência e da evolução sobre a COVID-19 e examinar o progresso no desenvolvimento de ferramentas eficazes da saúde. A cúpula virtual hospedou mais de 1000 pesquisadores e cientistas. Um dos resultados foi a 
concordância de que mais ensaios eram necessários para testar antivirais, drogas imunobiológicas e agentes antitrombóticos, bem como terapias combinadas, em diferentes estágios da doença (OMS, 2020).

No entanto, vários medicamentos foram sugeridos como potenciais terapias no primeiro ano da Pandemia, por isso essa revisão, dos estudos publicados no primeiro ano da pandemia, narra as estratégias terapêuticas. Os resultados da pesquisa evidenciaram que as terapêuticas à COVID-19 se apresentam em estratégias farmacológicas de três níveis de ações, a saber: alvos imunológicos; alvos baseados no vírus; alvo baseado no hospedeiro. Enquanto as estratégias não-farmacológicas são recursos que podem contribuir com o tratamento da doença sem envolver um alvo específico com uma molécula farmacológica.

\section{Estratégias Farmacológicas}

Notoriamente evidenciou uma gama diversificada das possibilidades terapêuticas que agem em diferentes alvos farmacológicos. Os principais foram os alvos imunológicos, alvos virais e alvos baseado no hospedeiro conforme o Quadro 1 (W. Liu et al., 2020).

Quadro 1 - Alvos terapêuticos e seus respectivos fármacos para o enfretamento da COVID-19, 2021.

\begin{tabular}{|c|c|c|c|c|c|}
\hline Alvos Imunológicos & & \multicolumn{2}{|c|}{ Alvos baseados no Vírus } & \multicolumn{2}{|c|}{ Alvos baseado no Hospedeiro } \\
\hline Fármaco & Ação Farmacológica & Fármaco & $\begin{array}{c}\text { Ação } \\
\text { farmacológica }\end{array}$ & Fármaco & $\begin{array}{c}\text { Ação } \\
\text { farmacológica }\end{array}$ \\
\hline $\begin{array}{l}\text { Células-tronco } \\
\text { Mesenquimais }\end{array}$ & Reparação do Tecido lesionado & $\begin{array}{l}\text { Lopinavir } \\
\text { Ritonavir }\end{array}$ & $\begin{array}{l}\text { Inibição da } \\
\text { 3CLpro }\end{array}$ & $\begin{array}{l}\text { Mivebresib } \\
\text { Bicalutamida } \\
\text { Apalutamida } \\
\text { Bromexina }\end{array}$ & $\begin{array}{l}\text { Inibidores da } \\
\text { TMPRSS2 }\end{array}$ \\
\hline $\begin{array}{l}\text { Timosina } \\
\text { Interferons } \\
\text { CYP-calcineurin- } \\
\text { NFAT } \\
\text { Ciclosporina }\end{array}$ & Supressão da resposta Imunológica & $\begin{array}{l}\text { Favipiravir } \\
\text { Galidesevir } \\
\text { BCX-4430 } \\
\text { Ribavirina } \\
\text { Remdesivir }\end{array}$ & Inibição da RdRp & $\begin{array}{l}\text { Dec-RVKR- } \\
\text { CMK } \\
\text { Agmatina } \\
\text { Andrografolide }\end{array}$ & $\begin{array}{l}\text { Inibidores } \\
\text { Furina }\end{array}$ \\
\hline $\begin{array}{l}\text { Plasma } \\
\text { Convalescente } \\
\text { CR3022 } \\
\text { Tociluzumabe } \\
\text { Fingolimode } \\
\text { Adalimumabe } \\
\text { Eculizumabe } \\
\text { Sarilumabe } \\
\text { Ixecizumabe } \\
\text { Meplazumabe }\end{array}$ & $\begin{array}{l}\text { Produção de anticorpos para combater a respostas } \\
\text { imunológicas }\end{array}$ & $\begin{array}{l}\text { Ivermectina } \\
\text { HE602 }\end{array}$ & Inibição do Hel & $\begin{array}{l}\text { Cloroquina } \\
\text { MLN-4760 } \\
\text { N-(2- } \\
\text { aminoetil)-1- } \\
\text { aziridina- } \\
\text { etanamina } \\
\text { (NAAE) } \\
\text { Glicirrizina }\end{array}$ & $\begin{array}{l}\text { Inibidores da } \\
\text { ECA2 }\end{array}$ \\
\hline & & LJ001 & $\begin{array}{c}\text { Inibição da } \\
\text { Endonuclease } \\
\text { Cap-dependente } \\
\text { Fotossensibilizador } \\
\text { do vírus } \\
\end{array}$ & $\begin{array}{l}\text { Clorpromazina } \\
\text { Ouabaína }\end{array}$ & $\begin{array}{l}\text { Inibidores da } \\
\text { Clatrina }\end{array}$ \\
\hline
\end{tabular}

Fonte: Elaborados pelos autores. 
Os potenciais alvos farmacológicos em nível viral foram os medicamentos que agem sobre as proteínas funcionais ligadas ao vírus SARS-CoV-2 como: a RNA-polimerase dependente-RNA (RdRp), protease viral (3CLpro); ATP-dependente helicase (Hel); Endonuclease Cap-dependentes. Os alvos farmacológicos ao nível do hospedeiro foi a proteína de membrana, ECA2, a Serina-serina protease transmembrana (TMSPSS2), Furina e Clatrina. E os alvos imunológicos basearam-se no reparo do tecido por meio de moléculas imunobiológicas para estimular o sistema imunológico (W. Liu et al., 2020).

Um dos maiores estudos de revisão conduzido por Seyedpour e colaboradores (2020) copilaram um total de 2.649 artigos identificados no PubMed, Web of Science, Scopus, EMBASE, Google Scholar e Biorxiv e obtiveram os seguintes resultados: foram identificados 22 estudos (um estudo in vivo e 21 estudos in vitro), e a glicoproteína de pico (também conhecida como proteína S ou spike) do SARS-CoV-2 foi o principal alvo de investigação em 19 estudos. As estratégias de terapia direcionadas contra os mecanismos de entrada do SARS-CoV-2 nas células se enquadram em quatro categorias principais: estratégias que visam os receptores de vírus no hospedeiro, estratégias de neutralização da proteína spike do SARSCoV-2, estratégias que visam a fusão do vírus e estratégias que visam os receptores endossômicos e outras vias que não sejam a entrada do vírus dependentes do endossoma.

Em uma revisão com metanálise avaliaram a associação entre a aplicação de Inibidores da Enzima Conversora da Angiotensina (IECA) e os Bloqueadores do Receptor da Angiotensina (BRAs) e o risco com a COVID-19 a nível de inflamação, gravidade da infecção e morte em pacientes. Essa revisão evidenciou que a terapia com IECA/BRAs pode estar associada ao fator inflamatório reduzido e à contagem elevada de células imunológicas (CD3, CD8). A metanálise não mostrou aumento significativo no risco de infecção por SARS-CoV-2 em pacientes recebendo terapia IECA/BRAs, porém foi associada a um risco reduzido da forma grave e mortalidade (X. Liu et al., 2020).

$\mathrm{Na}$ ausência de diretrizes de tratamento clinicamente comprovadas, vários medicamentos estão sendo reaproveitados clinicamente. Estes incluem antivirais (remdesivir, lopinavir/ritonavir, umifenovir e favipiravir), interferon, antimaláricos (cloroquina/ hidroxicloroquina), medicamentos antiparasitários (ivermectina e nitazoxanida), imunobiológicos (anticorpos monoclonais e antagonista do receptor de interleucinas), terapias celulares (células-tronco mesenquimais e células assassinas naturais, plasma convalescente e adsorvente de citocinas) (Samaddar et al., 2020; Zhao, 2020).

Embora vários estudos observacionais tenham afirmado que muitos desses agentes são eficazes, com base em suas atividades in vitro e experiências das epidemias de SARS e MERS, os dados atualmente disponíveis permanecem inconclusivos devido a critérios de seleção de pacientes mal definidos, tamanho pequeno da amostra, falta de controles simultâneos e uso de resultados intermediários (Samaddar et al., 2020; Nadaroglu, 2020).

Existe uma lista com vários ativos farmacológicos que podem ser utilizados terapeuticamente para a COVID-19, mas que ainda depende de ensaios clínicos para embasar diretrizes médicas (Andrade et al., 2020). Os fatores que levam à baixa qualidade dos resultados dos ensaios clínicos são a dificuldade de não conseguir uma amostra homogênea ou um grupo placebo sem intervenção. Sem nenhum balizador os resultados podem ser significativos na evidência clínica, porém as avaliações dos efeitos adversos tornam inseguro a prática clínica (Rafiee et al., 2021).

\section{Alvos imunológicos}

Baseia-se na pesquisa in vivo e in vitro de medicamentos imunobiológicos, terapia celular, anti-inflamatórios estoroidais e imunossupressores.

\section{Medicamentos Imunobiológicos}

$\mathrm{O}$ anticorpo monoclonal tocilizumab demonstrou uma tendência de associação para redução da mortalidade entre pacientes de UTI (Ip et al., 2020; González-gay et al., 2021). O tocilizumab é um inibidor da Interleucina-6, obteve sucesso 
para tratar a tempestade de citocinas provocada pela COVID-19 (Botond et al., 2020; Nasonov \& Samsonov, 2020). Apesar do pequeno tamanho da amostra e da natureza retrospectiva do trabalho, este resultado sugere fortemente que o tocilizumab pode reduzir o número de admissões na UTI e/ou mortalidade em pacientes com pneumonia grave por SARS-CoV-2 (Klopfenstein et al., 2020).

Relatórios iniciais compreendendo um estudo de 21 pacientes graves e críticos com a COVID-19 na China e um estudo na França apresentaram benefícios clínicos e segurança terapêutica com a utilização do tocilizumab, infliximabe ou adelimumabe (Feldmann et al., 2020).

Outro estudo com três pacientes, que observou a administração de eculizumab, um anti-C5, obteve um declínio acentuado nos Dímeros D e contagens de neutrófilos em todos os três casos, além da normalização das funções hepáticas e creatinina em dois pacientes (Laurence et al., 2020).

\section{Terapias Celulares}

Já uma revisão de 80 estudos apontou que 24 estudos forneceram dados de resultados clínicos sobre o uso de plasma convalescente (33 pacientes), anticorpos monoclonais (55 pacientes), interferon (31 pacientes), terapia com células-tronco mesenquimais ( 8 pacientes) e imunoglobulina (63 pacientes). Os resultados demonstraram, exceto por nove pacientes graves que morreram após o tratamento, que a maioria dos pacientes foram recuperados da COVID-19 com melhora nos sintomas clínicos e avaliação laboratorial (Mansourabadi et al., 2020).

O anticorpo baritinibe pode limitar a síndrome de liberação de citocinas associada a COVID-19 e pode ser útil porque atua contra uma ampla gama de citocinas (Cantini et al., 2020).

Os estudos evidenciados por Wang et al., (2020) remetem que, após a aplicação de IFN- $\alpha 2 b$ precocemente e o umifenovir isoladamente ou em conjunto, ocorre redução da mortalidade e recuperação acelerada em comparação com o tratamento isolado de lopinavir e ritonavir. Também concluiu que a administração de IFN- $\alpha 2 b$, durante o estágio inicial da COVID-19, pode induzir respostas clínicas favoráveis.

O tratamento com vesícula extracelular (EV) para Síndrome do Desconforto Respiratório Agudo (SDRA), tem se mostrado promissor, pois resultou na atenuação da inflamação (redução de citocinas pró-inflamatórias e infiltração de neutrófilos, polarização de macrófagos M2); regeneração do epitélio alveolar (diminuição da apoptose e estimulação da produção de surfactante); reparação da permeabilidade microvascular (aumento das proteínas da junção das células endoteliais); prevenção da fibrose (redução da produção de fibrina) (Khalaj et al., 2020).

Uma prática terapêutica, em nível imunológico, que foi amplamente discutida é a terapia com plasma convalescente, pois apresentou diminuição do risco de mortalidade, produção de anticorpos, redução da carga viral e o encurtamento do tempo da doença (Sun et al., 2020). Embora, o plasma convalescente não tenha encurtado o tempo de recuperação (Wiersinga et al., 2020).

A eficácia da terapia do plasma convalescente para a COVID-19 foi constatada numa revisão sistemática com metanálise, os quais obtiveram como desfechos primários uma melhora clínica, menores índices de mortalidade e tempo de internação. Aspectos positivos e negativos não suportam os benefícios da mortalidade, nem na melhora das condições clínicas e tempo de internação (Vegivinti et al., 2021; Langhi et al., 2020; Dassarma et al., 2021; Alijotas-reig et al., 2020; Nagoba et al., 2020).

Em um ensaio clínico, oito indivíduos com a forma leve da COVID-19 receberam, pelo menos, uma dose de transfusão de plasma convalescente e todos os indivíduos melhoraram. A contagem de linfócitos tendeu a aumentar, enquanto a lactato desidrogenase, a creatina quinase e a aspartato aminotransferase tenderam a diminuir. No entanto, a PCR aumentou temporariamente em três indivíduos. O tempo médio para que o teste de ácido nucléico com SDRA se tornasse negativo foi 2,5 
dias após a transfusão de plasma convalescente. Enquanto isso, a plasma convalescente provavelmente aumenta a resposta inflamatória ao SARS-CoV-2 temporariamente em pessoas com imunidade antiviral insuficiente. No entanto, os efeitos do plasma convalescente não são permanentes (Ji et al., 2021).

\section{Anti-inflamatórios estoroidais}

Além dos anticorpos, os anti-inflamatórios estoroidais também demonstraram resultados. Em um estudo randomizado com 2104 pacientes com COVID-19, a dexametasona reduziu a mortalidade de todas as caudas de mortes, e que associado ao remdesivir o tempo para recuperação foi menor, de 15 a 11 dias (Wiersinga et al., 2020). Mas, a utilização de antiinflamatórios corticosteroides requer cautela, pois há risco maior de inibição dos fatores de necrose tumoral, responsáveis pela diminuição das chances de infecção grave (Veenstra et al., 2020).

A dexametasona é o primeiro medicamento esteroide conhecido que pode salvar a vida de pacientes gravemente enfermos e foi demonstrado em um ensaio clínico randomizado, no Reino Unido, que reduziu a taxa de mortalidade em pacientes com COVID-19 (Trivedi et al., 2020; OMS, 2020).

Cabe ressaltar que boa parte das estratégias terapêuticas estão sendo extraídas das bases da terapia oncológica imunomoduladora. É o caso, da utilização de doses baixas de dexametasona que demonstrou benefícios às manifestações clínicas da COVID-19 (Parasher, 2020). Embora, desperta para vigilância sobre os efeitos indesejados do uso de antiinflamatórios estoroidais associados a hidroxicloroquina, principalmente aos efeitos cardiotóxicos (Campbell et al., 2020).

Outro corticosteroide, a metilprednisolona, foi utilizada por dois peruanos, que realizaram pulsos desta medicação em suas casas, devido à indisponibilidade de leitos, e se recuperaram da tempestade de citocinas (Sauñe et al., 2020).

No decorrer do primeiro ano da pandemia, outras evidências científicas foram obtidas num estudo experimental em 10 hospitais da China. Foi observado que o uso de corticosteroides pode atrasar a eliminação do SARS-CoV-2 de pacientes com a COVID-19 e, por isso, deve ser usado com cautela (Huang et al., 2020). Outra investigação científica não encontrou melhora na função respiratória ou do tempo de alta hospitalar associada ao uso de glicocorticoides em altas doses (RodríguezMolinero et al., 2021).

Os esteroides reduziram a mortalidade em pacientes com COVID-19 que precisam de oxigenioterapia de suporte ou ventilação mecânica invasiva, porém necessitam de monitoramento (Mattos-Silva et al., 2020).

Os resultados decepcionantes dos ensaios clínicos randomizados da terapia de corticosteroides ao longo do primeiro ano da pandemia da COVID-19 pode ter sido influenciado pela inconsistência do conhecimento da classificação clínica a respeito da sequência de três níveis de estágios de infecção do SARS-CoV-2. Com base na evidência de que, durante o curso clínico a COVID-19 progride de uma infecção viral para uma resposta super inflamatória de automanutenção, observado pelos resultados quase inesperados obtidos com terapia com corticosteroides, nos casos moderados, graves e críticos (de Simone \& Mancusi, 2020). Tais afirmações científicas contribuíram com resultados controversos do uso de corticosteroide e hidroxicloroquina (Razmi et al., 2020).

A classificação clínica dos três níveis dos estágios da doença (moderados, graves e críticos) atrelado ao início correto da terapia com corticosteroide foi proposta por Siddiqi e Mehra (2020), em que deve iniciar a partir do sexto dia após o início dos sintomas e perdurar até décimo sétimo dia (De Simone \& Mancusi, 2020).

Entretanto, a terapia com corticosteroides se mostrou efetiva e recomendada no tratamento em pacientes com COVID19 grave. Pois um estudo, que atribuiu 12.862 casos da COVID-19 em 21 hospitais na província de Hubei, evidenciou a diminuição da proporção de neutrófilos para linfócitos. É importante ressaltar que o tratamento com corticosteroides está associado a um menor risco de mortalidade por todas as causas. Por outro lado, em indivíduos com proporção menor de neutrófilos e leucócitos ou com diabetes tipo 2 não foi associado à redução da mortalidade, mas sim ao aumento do risco de 
hiperglicemia e infecções (Cai et al., 2021).

A terapia combinada de talidomida mais glicocorticoide em baixa dosagem foi eficaz em melhorar o prognóstico de pacientes com COVID-19 crítico. O mecanismo de ação é provavelmente a inibição da produção de citocinas inflamatórias (Y. Li et al., 2021).

\section{Imunossupressores}

A talidomida, imunossupressora, acelerou significativamente a redução da carga viral do SARS-CoV-2 e encurtou o tempo de internação hospitalar dos pacientes afetados, reduzindo a necessidade de ventilação mecânica em pacientes críticos com COVID-19 (Y. Li et al., 2021).

\section{Alvos baseados no vírus}

Os alvos farmacológicos baseados no vírus prospectaram esperanças falidas no primeiro ano da pandemia. Estes medicamentos foram utilizados e desaconselhados na medida em que novos estudos eram publicados. Mesmo assim, foram usados de forma generalizada, desde prescrições médicas até na forma automedicada. Entre os medicamentos pesquisados estão os antivirais, antiparasitários e antibióticos.

\section{Antivirals}

Em suma, uma revisão publicada na base científica Cochrane, em dezembro de 2020, destaca os medicamentos umifenovir, remdesivir e o favipiravir como agentes antivirais mais promissores, por melhorar a saúde dos pacientes infectados (Trivedi et al., 2020).

No estudo que cita os resultados de três outros estudos, quando avaliados a combinação da cloroquina com remdezevir ou Interferon produziram a inibição da replicação do SARS-CoV-2. (Shereen et al., 2020; Zhaori et al., 2020) ou, somente a associação com remdesevir (Carvajal et al., 2020).

O remdesivir atenua a progressão da doença, levando a menor chance de ventilação mecânica ou oxigenação extracorpórea, oferecendo alta hospitalar para pacientes com COVID-19. No entanto, o remdesivir não afeta as taxas de mortalidade (Charan et al., 2021).

Os estudos evidenciados por Wang et al., (2020) após a aplicação de umifenovir isoladamente, ocorre redução da mortalidade e recuperação acelerada em comparação com o tratamento isolado de lopinavir e ritonavir.

A cloroquina, remdesivir, lopinavir/ritonavir ou ribavirina têm sido bem-sucedidos na inibição da SARS-CoV-2 in vitro. Os resultados iniciais de uma série de ensaios clínicos envolvendo vários protocolos de administração de cloroquina apontam principalmente para o seu efeito benéfico. No entanto, eles podem não ser eficazes na viremia persistentemente elevada, enquanto os resultados com ivermectina ainda não estão disponíveis. Curiosamente, a azitromicina, um antibiótico macrolídeo em combinação com hidroxicloroquina, pode produzir benefícios clínicos como um adjuvante (Drożdżal et al., 2020). Em 04 de julho a OMS anunciou o declínio dos estudos do projeto Solidarity com os medicamentos lopinavir / ritonavir, devido a ausência de benefício clínico em termos de redução da mortalidade (OMS, 2020).

O estudo de Yao et al., (2020) buscou apresentar a eficácia do lopinavir in vivo e in vitro nas epidemias de SARS e MERS para complementar as evidências terapêuticas na COVID-19. O tratamento de pacientes com SARS-CoV-2 com lopinavir melhorou seus resultados clínico.

Atualmente, há evidências insuficientes de eficácia do remdesivir e outros antivirais no tratamento ou prevenção de COVID-19, pois os resultados terapêuticos são ínfimos aos efeitos adversos de arritmia cardíaca com risco em pacientes com doença cardiovasculares preexistentes (Jha et al., 2020; Gollob, 2020). 
No caso da terapia com ribavirina, o estudo de coorte retrospectivo, conduzido por Tong et al., (2020) desqualifica a terapia em casos graves da COVID-19 por não estar associada à melhora do tempo de conversão negativa para o teste SARSCoV-2 e não está associada a uma taxa de mortalidade melhorada.

O estudo publicado por Bassetti et al., (2020) discutiu uma lista de 8 questões terapêuticas práticas, a fim de considerar que muitos medicamentos antivirais e anti-inflamatórios off-label estavam sendo administrados. Eles não desencorajam seus usos, mas incubem aos médicos estar cientes de que, devido à falta de evidências de alto nível, eles podem ser eticamente justificáveis apenas em pacientes com piora, improváveis de melhorar com apenas cuidados de suporte e que não podem ser inscritos nos ensaios clínicos randomizados (RCTs). A implementação de RCTs bem projetados deve ser expandida tanto quanto possível, pois os RCTs são a maneira mais segura de mudar para melhor nossa abordagem aos pacientes COVID-19, incluindo nossas opiniões de primeira linha.

Outro estudo que apresentou resultados positivos sugere uma terapia de combinação quádrupla (ribavirin, lopinavir, ritonavir, umifenovir) pois após o tratamento, a coagulação anormal e os leucócitos melhoraram significativamente com um melhor prognóstico (X. Li et al., 2020). Sobre os aspectos de inibição viral, o lopinavir e o ritonavir não apresentaram diferenças significativas no regime de tratamento padrão da forma grave da doença COVID-19 (Yousefifard et al., 2020).

\section{Antibióticos}

Um estudo in-silico demonstrou segurança e efetividade as terapias lisossomotrópicas e drogas direcionadas a lissossomas que estão em uso clínico, baixo custo e ampla disponibilidade, como por exemplo a azitromicina (Homolak \& Kodvanj, 2020). No estudo de coorte observacional sugere que a hidroxicloroquina em combinação com azitromicina, não obteve benefício de sobrevida entre pacientes com COVID-19 hospitalizados (Ip et al., 2020).

Um estudo controle realizado, em que 64,6\% dos pacientes usaram hidroxicloroquina associada a azitromicina, constatou o aumento do tempo de prolongamento de frequências cardíacas quando comparadas às análises durante o prétratamento (Seyhan et al., 2020).

\section{Antiparasitários}

A monoterapia com hidroxicloroquina (HCQ) de baixa dosagem foi independentemente associada a uma mortalidade mais baixa em pacientes hospitalizados, diagnosticados e tratados precocemente ou posteriormente após o início dos sintomas da COVID-19 (Catteau et al., 2020).

O estudo de coorte observacional sugere que a HCQ não foi associada a um benefício de sobrevida entre pacientes graves hospitalizados (Ip et al., 2020).

Em pacientes ambulatoriais adultos com infecção moderada por COVID-19, a eficácia de febuxostat e HCQ não foi diferente em termos de resolução das manifestações clínicas, exames laboratoriais e dos resultados das tomografias computadorizadas pulmonares (Davoodi et al., 2020).

Os efeitos colaterais raros de HCQ que eram de menor importância podem ganhar maior relevância com aplicação em toda a população. Esses efeitos colaterais raros incluem retinopatia, prolongamento do intervalo QT, risco à morte cardíaca súbita por arritmias malignas (Stremmel et al., 2020).

A falha da HCQ em mostrar eliminação viral ou benefícios clínicos com efeitos adversos adicionais superam seu efeito protetor da progressão radiológica em pacientes não graves com a COVID-19 (Elsawah et al., 2021).

Um estudo controle realizado com 35,4\% de pacientes infectados com a COVID-19 sob tratamento com monoterapia hidroxicloroquina, constatou o aumento do tempo de prolongamento de frequências cardíacas quando comparadas às análises durante o pré-tratamento (Seyhan et al., 2020). Um estudo de revisão, elaborado do Jankowska et al., (2020), avaliou o 
desenho de 04 estudos multicêntricos, com ou sem grupo controle, com diferentes quantidades de amostras, e constatou um desfecho não favorável, tanto em índices de mortalidade, internação quando foi utilizado tratamento baseados com HCQ, evidenciou também aumentos dos efeitos colaterais provocados por esses medicamentos.

Em um estudo com amostra de 440 pacientes, divididos em grupos de alta e baixa dosagem de cloroquina, observouse que a alta dosagem de cloroquina não deve ser recomendada para pacientes gravemente enfermos com a COVID-19 por causa de seus riscos potenciais à segurança, especialmente quando administrada simultaneamente com azitromicina e oseltamivir. Esses achados não podem ser extrapolados para pacientes com a forma leve da COVID-19 (Borba et al., 2020).

Numa revisão de 12 estudos observacionais e 03 estudos randomizados que incluíram 10.659 pacientes, dos quais 5.713 receberam HCQ e 4.966 receberam apenas o tratamento padrão, a eficácia de HCQ para COVID-19 foi inconsistente entre os estudos. A metanálise dos estudos incluídos não revelou redução significativa na mortalidade com o uso de HCQ, tempo para controlar a febre ou deterioração clínica e desenvolvimento de SDRA com HCQ. Houve um risco maior de anormalidades e arritmia de ECG com HCQ (Elavarasi et al., 2020).

Atualmente, não há evidências suficientes de eficácia da hidroxicloroquina no tratamento ou prevenção de COVID19, pois os resultados terapêuticos são ínfimos aos efeitos adversos do prolongamento do intervalo de QT e arritmia cardíaca com risco em pacientes com doença cardiovasculares preexistentes (JHA et al., 2020; GOLLOB, 2020). Em outros cincos ensaios clínicos randomizados a hidroxicloroquina mostrou ser consistentemente ineficaz e insegura no tratamento da COVID19 (Zhaori et al., 2020). Relata-se efeitos cardiotóxicos quando combinados terapias de corticoides e HCQ (Campbell et al., 2020).

Nenhuma diferença significativa foi encontrada em termos de taxas de uso de hidroxicloroquina ou colchicina entre aqueles que foram considerados positivos para SARS-CoV-2 e aqueles que foram negativos (Gendelman et al., 2020).

Em 17 de junho de 2020 a OMS anunciou que o estudo da hidroxicloroquina sob organização do projeto Solidarity Trial estava sendo interrompido para a COVID-19. A decisão foi baseada em evidências randomizadas em larga escala dos ensaios, revisões de evidências publicadas e disponíveis de outras fontes, que mostraram que a hidroxicloroquina não reduziu a mortalidade para pacientes com COVID-19 hospitalizados (OMS, 2020).

Em 15 de outubro de 2020 a OMS anunciou evidências conclusivas sobre a eficácia dos medicamentos reutilizados para COVID-19. Os resultados provisórios do Solidarity Trial indicaram que os regimes de remdesivir, hidroxicloroquina, lopinavir / ritonavir e interferon pareceram ter pouco ou nenhum efeito na mortalidade em 28 dias ou no curso intra-hospitalar da COVID-19 entre pacientes hospitalizados (OMS, 2020).

Em um cenário promissor em que há diagnóstico, tratamento e acompanhamento precoce, como observado no estudo publicado em janeiro de 2021, quando pacientes foram atendidos por telemedicina, os resultados obtidos são positivos. O estudo foi conduzido por Procter et al., (2021), e verificou os resultados numa amostra de pacientes com idade de 50,5 $\pm 13,7$ anos, destes 61,6\% eram mulheres com risco moderado ou alto para COVID-19, que receberam tratamento empírico via telemedicina. Pelo menos dois agentes com atividade antiviral contra SARS-CoV-2 (zinco, hidroxicloroquina, ivermectina) e um antibiótico (azitromicina, doxiciclina, ceftriaxona) foram usados junto com budesonida inalada e/ou intramuscular e com dexametasona no tratamento inicial da COVID-19. Para os pacientes com sintomas de alta gravidade, foi realizada administração urgente de albuterol por nebulização, budesonida inalada e expansão do volume intravenoso com tiamina parenteral, sulfato de magnésio, ácido fólico, vitamina B12. Um total de 320 (34,7\%) foram tratados, resultando em $6(1,9 \%)$ e $1(0,3 \%)$ dos pacientes que foram hospitalizados e morreram, respectivamente. $\mathrm{O}$ estudo concluiu que os atendimentos ambulatoriais precoces, associados a tratamentos com multidroga são seguros, viáveis e estão associados a baixas taxas de hospitalização e morte.

Sobre a variedade de medicamentos utilizados empiricamente no problema de saúde que assola o mundo moderno, 
estudos despontam as consequências negativas da utilização demasiada e irracional dos medicamentos antimaláricos, antivirais, antibióticos, anticorpos, anticoagulante. (Najar Nobari et al., 2020).

Em 20 de novembro de 2020 a OMS publicou uma diretriz sobre terapêutica e COVID-19, com novas informações para os médicos, incluindo uma recomendação condicional contra o uso de remdesivir em pacientes hospitalizados com COVID-19, independentemente da gravidade da doença (OMS, 2020).

\section{Alvos baseados no hospedeiro}

Os alvos baseados no hospedeiro são terapias sistêmicas utilizadas para combater os sintomas e a infecção pelo SARS-CoV-2. A molécula farmacológica, conhecida como anakinra (inibidor dos receptores da IL-1) evitou a necessidade da ventilação mecânica em pacientes com pneumonia por COVID-19 grave, acelerou o desmame da oxigenioterapia e acelerou a transição do paciente para o ar ambiente, além de reduzir significativamente os biomarcadores de inflamação. Os resultados do estudo não demonstraram diferença significativa na mortalidade intra-hospitalar. Este estudo forneceu evidências adicionais para a utilidade de anakinra na pneumonia por COVID-19 grave (Balkhair et al., 2021).

O arbidol, um inibidor de fusão de membrana aprovado para o vírus da gripe, está atualmente em testes clínicos contra COVID-19. Neste contexto, alguns análogos restringiram a interação do SARS-CoV-2 com a ECA2 e as proteases furina e TMPRSS2 do hospedeiro (Choudhary \& Silakari, 2020). Um estudo conduzido por Xu et al., (2020a) infere que a terapia com arbidol combinado com IFN- $\alpha 2 b$ pode ser usada como um método eficaz para melhorar a pneumonia por COVID-19 em pacientes leves, embora seja impotente com a aceleração da eliminação do vírus.

Os estudos de Lovetrue (2020) e Hamizi et al. (2020) defendem protocolos citotóxicos, combinando o irinotecano e etoposídeo (inibidores da topoisomerase I e II, respectivamente) e etoposídeo com dexametasona. O esquema terapêutico poderia ser um tratamento excepcionalmente eficaz para proteger pacientes em estado crítico de morte causada por tempestades de citocinas específicas da COVID-19 desencadeadas por sepse, SDRA e outras comorbidades fatais.

O tratamento para trombose nos casos leves e graves inclui a terapia anticoagulação durante a internação hospitalar e também no tratamento domiciliar pós-COVID-19 (Poor et al., 2020). Os autores Pierini et al., (2020); Shi et al., (2021); Alijotas-Reig et al., (2020) acreditam que a causa das coagulopatias são decorrentes do dano vascular sistêmico diretamente determinado pelo vírus no endotélio, o qual expressa o quadro trombótico, necessitando da utilização dos antitrombóticos, como a heparina de baixo peso molecular. Mas afirma, que ainda as recomendações de anticoagulantes precisam passar por ensaios clínicos.

A terapia antitrombótica pré-admissão, tanto antiplaquetária quanto anticoagulante, não parece mostrar efeito protetor nas formas graves da COVID-19 com SDRA evoluindo rapidamente para óbito (Russo et al., 2020). O estudo de Lawlor et al., (2021) apresenta discordância no tempo de tromboplastina parcial ativada e níveis de anti-fator Xa em pacientes com COVID19 em terapia com heparina intravenosa fracionada.

Uma combinação terapêutica entre heparina e nafamostate mostrou eficácia, incluindo os efeitos da antitrombina, antiplasmina e antitripsina. Houve melhora do paciente do caso relatado, utilizando nafamost contra a COVID-19, cuja hipoxemia grave, foi provavelmente causada por coagulação disseminada e embolia pulmonar. Além disso, são discutidos os mecanismos benéficos do nafamostat contra COVID-19 e a necessidade de atenção à hipercalemia como efeito adverso (Takahashi et al., 2021).

$\mathrm{Na}$ análises dos resultados desta revisão, observou-se alguns estudos aleatórios como: A apelina pode suprimir ECA melhorando a inflamação, trombose e vasoconstrição mediada por Angiotensina II em pacientes com a COVID-19 (Saeedi Saravi \& Beer, 2020); O colecalciferol reduziu significativamente as admissões na unidade de terapia intensiva (Entrenas Castillo et al., 2020); As estatinas não melhoraram os resultados hospitalares de pacientes com infecções por COVID-19 
(Hariyanto \& Kurniawan, 2020); E os estimuladores de colônias granulócitos de (G-CSF) pioraram rapidamente a neutropenia após a administração (Taha et al., 2020).

Alguns estudos referentes os benefícios da fitoterapia foram encontrados, como: no artigo desenvolvido por Muchtaridi et al., (2020), que evidenciou resultados positivos de compostos naturais, com base na ação de flavonoides, por interagir com a ECA2 e combater a COVID-19. Esta postulação pode ser de interesse porque esses compostos também apresentam atividade antiviral in vitro. Em outro estudo, destacou-se a importância de plantas com propriedades farmacológicas que podem auxiliar a minimizar os efeitos do SARS-CoV-2 (Oladele et al., 2020).

A nanocurcumina, como um agente anti-inflamatório à base de ervas, pode ser capaz de modular a resposta das citocinas inflamatórias, especialmente a expressão de mRNA de IL-1 $\beta$ e IL-6 e a secreção de citocinas em pacientes com COVID-19, o que pode causar uma melhora clínica e recuperação geral (Valizadeh et al., 2020).

Ensaios clínicos randomizados, realizadas no início da pandemia, evidenciaram efeitos significativos da combinação com a fitoterapia e medicina ocidental, pois melhoraram a febre, tosse, escarro, inflamação na orofaringe, fadiga, embora os riscos de viés dos estudos não foram bem definidos (Ang et al., 2020). Sobre a ausência de ensaios clínicos randomizados, não há atualmente nenhuma evidência confiável sobre os efeitos e as intervenções terapêuticas contra a COVID-19 na utilização de medicamentos fitoterápicos chineses (López-Alcalde et al., 2020).

\section{Estratégias não farmacológicas}

No estudo de revisão de um órgão público de saúde Essald (2020) mensurou as evidências terapêuticas da ozonioterapia, que após testada in vitro, apresentou baixa qualidade de evidências para inativar vírus ou induzir a expressão de enzimas antioxidantes e citocinas (IFN- $\delta$, TNF- $\alpha$ e IL-4) relacionados à imunidade celular.

A pneumonia por COVID-19 desenvolve um processo celular de estresse oxidativo que parece ser altamente prejudicial para os tecidos pulmonares. Embora a inalação do gás ozônio tenha se mostrado tóxico para os pulmões, as evidências recentes sugerem que sua administração por vias apropriadas e em pequenas doses pode, paradoxalmente, induzir uma reação adaptativa capaz de diminuir o estresse oxidativo endógeno. A terapia com ozônio é recomendada para combater os efeitos perturbadores da COVID-19 grave nos tecidos pulmonares (Izadi et al., 2020; Franzini et al., 2020; Shah et al., 2021).

Ensaios clínicos randomizados evidenciaram resultados significativos da combinação com a fitoterapia e medicina ocidental, pois melhoraram a sintomatologia inicial da doença como a febre, tosse, escarro, inflamação na orofaringe, fadiga (Ang et al., 2020).

Uma revisão sistemática e metanálise interpretou os resultados do tratamento combinado da COVID-19 com a medicina chinesa e ocidental, sendo este eficaz no controle dos sintomas e na redução da taxa de progressão da doença (Luo et al., 2020). A medicina tradicional chinesa mostrou-se conflituosa a respeito dos benefícios seguros e eficazes para a COVID19 (S. Li et al., 2020).

Quase metade dos pacientes com COVID-19 recebendo Ventilação Mecânica Invasiva (VMI) morreram com base na taxa de letalidade relatado pelo estudo, maior em pacientes mais velhos e nos primeiros epicentros da pandemia, o que pode ser influenciado pelos recursos limitados da UTI (Lim et al., 2021). Os desfechos dos grupos de pacientes com a COVID-19 submetidos a VMI que preencheram os critérios dos procedimentos de oxigenioterapia por membrana extracorpórea não foram satisfatórios quando comparados entre os grupos, pois associou aumento do tempo de internamento na UTI, nos marcadores de coagulação, na inflamação e lesão cardíaca, além de morte (Alnababteh et al., 2020).

A oxigenioterapia, uma estratégia não farmacológica em pacientes com pneumonia por COVID-19 obteve melhoras absolutas na média da saturação de oxigênio um dia após a radioterapia (Kirsch, 2020). 
Um estudo observacional prospectivo, avaliou a utilização da oxigenioterapia nasal de alto fluxo em momentos diferentes nas posições de pronação e não encontrou redução do risco da intubação, mas um atraso na intubação em comparação com os pacientes que estavam sendo tratados com oxigenioterapia nasal de alto fluxo sozinho (Ferrando et al., 2020). O uso de terapia de oxigênio nasal de alto fluxo está associado a uma redução na taxa de ventilação mecânica invasiva e mortalidade geral em pacientes com infecção por COVID-19 (Patel et al., 2020; Vianello et al., 2020; Jha et al., 2020)

Para além das práticas não farmacológicas as observações apontadas no estudo de Dixit (2020) inclui as práticas do exercício físico no fortalecimento do sistema imunológico, abordando para uma melhor defesa imunológica contra a COVID19.

\section{Considerações Finais: Ainda não Chegamos no Final da História}

Entre as estratégias terapêuticas farmacológicas utilizadas no primeiro ano da pandemia da COVID-19 destacaram-se os medicamentos antirretrovirais, antibióticos, antiparasitários, anti-inflamatórios estoroidais, anticorpos monoclonais, terapia celular, imunossupressores e anticoagulantes. Já as estratégias terapêuticas não-farmacológicas basearam se na medicina chinesa, oxigenioterapia/ ventilação mecânica, infusões e chás com plantas, exercícios físicos, ozonioterapia.

As estratégias terapêuticas farmacológicas incidiram-se em três níveis: alvos imunológicos e/ou processos de sinalização imunológicas como a utilização de imunossupressão com anticorpos, anticitocinas, plasma convalescentes, célulastronco mesenquimais e vitamina D. Os alvos baseados em hospedeiro, buscaram evidenciar resultados terapêuticos de agentes capazes de interagir nos receptores celulares e enzimas envolvidas no processo de infecção do vírus, como a TMSPSS2, furina, ECA2 e Clatrina através dos medicamentos, anakinra, anticoagulantes, antineoplásicos, bicalutaminda, apalutamida, arbidol. Os alvos baseados no vírus buscaram a inibição das proteínas virais funcionais como 3CLpro; RdRp; Hel, a partir dos antivirais ritonavir, favipiravir, lopinavir, ribavarina, remdesivir e de antiparasitários hidroxicloroquina e ivermectina.

As estratégias terapêuticas não-farmacológicas são recursos que podem contribuir com o tratamento da doença sem envolver um alvo específico com uma molécula farmacológica. Como por exemplo: ozonioterapia, oxigenioterapia, exercício físico, medicina tradicional chinesa, ventilação mecânica invasiva.

Essa revisão narrativa trouxe os constructos terapêuticos da COVID-19 ao longo do primeiro ano da pandemia. Logo, conhecemos os resultados publicados da eficácia terapêutica dos medicamentos reaproveitados, haja vista, que não se desenvolveu nenhuma fórmula farmacêutica inovadora para o tratamento terapêutico específico para essa morbidade, neste período. Observou-se que no primeiro ano de Pandemia a conduta do uso off-label foi a mais usada, uma vez que não há um medicamento exclusivo para a COVID-19. Assim, mais estudos necessitam ser desenvolvidos para um desfecho de uma história vitoriosa conta a COVID-19.

Os estudos mais indicados para melhor evidenciar as terapias eficazes são ensaios clínicos randomizados e controlados com diferentes perfis populacionais em fases fisiopatológicas distintas da COVID-19. Estudos de revisões sistemáticas com metanálise também são importantes para embasar novas políticas públicas de saúde.

\section{Referências}

Alijotas-Reig, J., Esteve-Valverde, E., Belizna, C., Selva-O’Callaghan, A., Pardos-Gea, J., Quintana, A., Mekinian, A., Anunciacion-Llunell, A., \& Miró-Mur, F. (2020). Immunomodulatory therapy for the management of severe COVID-19. Beyond the anti-viral therapy: A comprehensive review. Autoimmunity Reviews, 19(7), 102569. https://doi.org/10.1016/j.autrev.2020.102569

Alnababteh, M., Hashmi, M., Drescher, G., Vedantam, K., Kohli, A., Hayat, F., Chopra, R., Oweis, E., \& Zaaqoq, A. (2020). Extracorporeal Membrane Oxygenation As Rescue Therapy for COVID-19 Induced Hypoxia: Single-Center Study. Chest, 158(4), A2411. https://doi.org/10.1016/j.chest.2020.09.010

Andrade, B. S., Rangel, F. de S., Santos, N. O., Freitas, A. dos S., Soares, W. R. de A., Siqueira, S., Barh, D., Góes-Neto, A., Birbrair, A., \& Azevedo, V. A. de C. (2020). Repurposing Approved Drugs for Guiding COVID-19 Prophylaxis: A Systematic Review. Frontiers in Pharmacology, 11(December), 1-12. https://doi.org/10.3389/fphar.2020.590598 
Balkhair, A., Al-Zakwani, I., Al Busaidi, M., Al-Khirbash, A., Al Mubaihsi, S., BaTaher, H., Al Aghbari, J., Al Busaidi, I., Al Kindi, M., Baawain, S., Al Alawi, A., Al Lawati, A., Al Rawahi, B., Al-Baimani, K., Al Zidi, K., Elfatih, N., Dawud, B., John, B., Rehman, F., ... Balkhair, O. (2021). Anakinra in hospitalized patients with severe COVID-19 pneumonia requiring oxygen therapy: Results of a prospective, open-label, interventional study. International Journal of Infectious Diseases, 103, 288-296. https://doi.org/10.1016/j.ijid.2020.11.149

Bassetti, M., Giacobbe, D. R., Aliberti, S., Barisione, E., Centanni, S., De Rosa, F. G., Di Marco, F., Gori, A., Granata, G., Mikulska, M., Petrosillo, N., Richeldi, L., Santus, P., Tascini, C., Vena, A., Viale, P., \& Blasi, F. (2020). Balancing evidence and frontline experience in the early phases of the COVID-19 pandemic: current position of the Italian Society of Anti-infective Therapy (SITA) and the Italian Society of Pulmonology (SIP). Clinical Microbiology and Infection, 26(7), 880-894. https://doi.org/10.1016/j.cmi.2020.04.031

Borba, M. G. S., Val, F. F. A., Sampaio, V. S., Alexandre, M. A. A., Melo, G. C., Brito, M., Mourão, M. P. G., Brito-Sousa, J. D., Baía-da-Silva, D., Guerra, M. V. F., Hajjar, L. A., Pinto, R. C., Balieiro, A. A. S., Pacheco, A. G. F., Santos, J. D. O., Naveca, F. G., Xavier, M. S., Siqueira, A. M., Schwarzbold, A., .. Lacerda, M. V. G. (2020). Effect of High vs Low Doses of Chloroquine Diphosphate as Adjunctive Therapy for Patients Hospitalized With Severe Acute Respiratory Syndrome Coronavirus 2 (SARS-CoV-2) Infection: A Randomized Clinical Trial. JAMA Network Open, 3(4), e208857. https://doi.org/10.1001/jamanetworkopen.2020.8857

Botond, L., László, G., Eniko, G., Alexandra, R., Zsuzsa, V., Éva, N., Eszter, M., Gabriella, B., Ilona, B., Péter, R., János, S., János, S., \& István, V. N. (2020). Anti-cytokine therapy in novel coronavirus disease (COVID-19) - The first administration of tocilizumab in Hungary at a department of infectology. Orvosi Hetilap, 161(26), 1070-1077. https://doi.org/10.1556/650.2020.31899

Cai, J., Li, H., Zhang, C., Chen, Z., Liu, H., Lei, F., Qin, J. J., Liu, Y. M., Zhou, F., Song, X., Zhou, J., Zhao, Y. C., Wu, B., He, M., Yang, H., Zhu, L., Zhang, P., Ji, Y. X., Zhao, G. N., ... Li, H. (2021). The Neutrophil-to-Lymphocyte Ratio Determines Clinical Efficacy of Corticosteroid Therapy in Patients with COVID-19. Cell Metabolism, 33(2), 258-269.e3. https://doi.org/10.1016/j.cmet.2021.01.002

Campbell, C. M., Guha, A., Haque, T., Neilan, T. G., \& Addison, D. (2020). Repurposing Immunomodulatory Therapies against Coronavirus Disease 2019 (COVID-19) in the Era of Cardiac Vigilance: A Systematic Review. Journal of Clinical Medicine, 9(9), 2935. https://doi.org/10.3390/jcm9092935

Cantini, F., Niccoli, L., Matarrese, D., Nicastri, E., Stobbione, P., \& Goletti, D. (2020). Baricitinib therapy in COVID-19: A pilot study on the safety and clinical impact. Journal of Infection, 81(2), 318-356. https://doi.org/10.1016/j.jinf.2020.04.017

Catteau, L., Dauby, N., Montourcy, M., Bottieau, E., Hautekiet, J., Goetghebeur, E., van Ierssel, S., Duysburgh, E., Van Oyen, H., Wyndham-Thomas, C., Van Beckhoven, D., Bafort, K., Belkhir, L., Bossuyt, N., Caprasse, P., Colombie, V., De Munter, P., Deblonde, J., Delmarcelle, D., ... Willems, E. (2020). Lowdose hydroxychloroquine therapy and mortality in hospitalized patients with COVID-19: a nationwide observational study of 8075 participants. International Journal of Antimicrobial Agents, 56(4). https://doi.org/10.1016/j.jiantimicag.2020.106144

Charan, T. R. V., Pederson, J. M., Saravu, K., Gupta, N., Barrett, A., Davis, A. R., Kallmes, K. M., \& Evanson, K. W. (2021). Remdesivir therapy in patients with COVID-19: A systematic review and meta-analysis of randomized controlled trials. Annals of Medicine and Surgery, 62(December 2020), 43-48. https://doi.org/10.1016/j.amsu.2020.12.051

Chen, N., Zhou, M., Dong, X., Qu, J., Gong, F., Han, Y., Qiu, Y., Wang, J., Liu, Y., Wei, Y., Xia, J., Yu, T., Zhang, X., \& Zhang, L. (2020). Epidemiological and clinical characteristics of 99 cases of 2019 novel coronavirus pneumonia in Wuhan, China: a descriptive study. The Lancet, 395(10223), 507-513. https://doi.org/10.1016/S0140-6736(20)30211-7

Choudhary, S., \& Silakari, O. (2020). Scaffold morphing of arbidol (umifenovir) in search of multi-targeting therapy halting the interaction of SARS-CoV-2 with ACE2 and other proteases involved in COVID-19. Virus Research, 289(July), 198146. https://doi.org/10.1016/j.virusres.2020.198146

Cueto, M. (2020). Os historiadores e as epidemias na América Latina Nas. História, Ciências, Saúde-Manguinhos, 27(4), 11028-11029. https://doi.org/10.23925/1806-9029.2018v30i2p1-4

Dassarma, B., Tripathy, S., \& Matsabisa, M. (2021). Emergence of ancient convalescent plasma (CP) therapy: To manage COVID-19 pandemic. Transfusion Clinique et Biologique, 28(1), 123-127. https://doi.org/10.1016/j.tracli.2020.11.004

Davoodi, L., Abedi, S. M., Salehifar, E., Alizadeh-Navaei, R., Rouhanizadeh, H., Khorasani, G., \& Hosseinimehr, S. J. (2020). Febuxostat therapy in outpatients with suspected COVID-19: A clinical trial. International Journal of Clinical Practice, 74(11), 1-8. https://doi.org/10.1111/ijcp.13600

de Simone, G., \& Mancusi, C. (2020). Finding the right time for anti-inflammatory therapy in COVID-19. International Journal of Infectious Diseases, 101, 247-248. https://doi.org/10.1016/j.ijid.2020.09.1454

Dixit, S. (2020). Can moderate-intensity aerobic exercise be an effective and valuable therapy in preventing and controlling the pandemic of COVID-19? Medical Hypotheses, 143(April). https://doi.org/10.1016/j.mehy.2020.109854

Drożdżal, S., Rosik, J., Lechowicz, K., Machaj, F., Kotfis, K., Ghavami, S., \& Łos, M. J. (2020). FDA-approved drugs with pharmacotherapeutic potential for SARS-CoV-2 (COVID-19) therapy. Drug Resistance Updates, 53(July), 100719. https://doi.org/10.1016/j.drup.2020.100719

Elavarasi, A., Prasad, M., Seth, T., Sahoo, R. K., Madan, K., Nischal, N., Soneja, M., Sharma, A., Maulik, S. K., Shalimar, \& Garg, P. (2020). Chloroquine and Hydroxychloroquine for the Treatment of COVID-19: a Systematic Review and Meta-analysis. Journal of General Internal Medicine, 35(11), 3308-3314. https://doi.org/10.1007/s11606-020-06146-w

Elsawah, H. K., Elsokary, M. A., Elrazzaz, M. G., \& Elshafie, A. H. (2021). Hydroxychloroquine for treatment of nonsevere COVID-19 patients: Systematic review and meta-analysis of controlled clinical trials. Journal of Medical Virology, 93(3), 1265-1275. https://doi.org/10.1002/jmv.26442

Entrenas Castillo, M., Entrenas Costa, L. M., Vaquero Barrios, J. M., Alcalá Díaz, J. F., López Miranda, J., Bouillon, R., \& Quesada Gomez, J. M. (2020). "Effect of calcifediol treatment and best available therapy versus best available therapy on intensive care unit admission and mortality among patients hospitalized for COVID-19: A pilot randomized clinical study." Journal of Steroid Biochemistry and Molecular Biology, 203(July). https://doi.org/10.1016/j.jsbmb.2020.105751 
ESSALD. (2020). Ozonoterapia para o tratamento de pacientes adultos com COVID-19. Ara Ii, 1-16.

Feldmann, M., Maini, R. N., Woody, J. N., Holgate, S. T., Winter, G., Rowland, M., Richards, D., \& Hussell, T. (2020). Trials of anti-tumor necrosis factor therapy for COVID-19 are urgently needed. The Lancet, 395(10234), 1407-1409. https://doi.org/10.1016/S0140-6736(20)30858-8

Ferrando, C., Mellado-Artigas, R., Gea, A., Arruti, E., Aldecoa, C., Adalia, R., Ramasco, F., Monedero, P., Maseda, E., Tamayo, G., Hernández-Sanz, M. L., Mercadal, J., Martín-Grande, A., Kacmarek, R. M., Villar, J., \& Suárez-Sipmann, F. (2020). Awake prone positioning does not reduce the risk of intubation in COVID-19 treated with high-flow nasal oxygen therapy: A multicenter, adjusted cohort study. Critical Care, 24(1), 1-11. https://doi.org/10.1186/s13054-02003314-6

Franzini, M., Valdenassi, L., Ricevuti, G., Chirumbolo, S., Depfenhart, M., Bertossi, D., \& Tirelli, U. (2020). Oxygen-ozone (O2-O3) immunochemical therapy for patients with COVID-19. Preliminary evidence was reported. International Immunopharmacology, 88(August), 106879. https://doi.org/10.1016/j.intimp.2020.106879

Garcia, L. P., \& Duarte, E. (2020). Intervenções não farmacológicas para o enfrentamento à epidemia da COVID-19 no Brasil. Epidemiologia e Servicos de Saude : Revista Do Sistema Unico de Saude Do Brasil, 29(2), 1-4. https://doi.org/10.5123/S1679-49742020000200009

Gendelman, O., Amital, H., Luigi, N., \& Watad, A. (2020). Continuous hydroxychloroquine or colchicine therapy does not prevent infection with SARS-CoV2: Insights from a large healthcare database analysis. Autoimmunity Reviews Journal, 19(January), 1-4.

Gollob, M. H. (2020). COVID-19, Clinical Trials, and QT-Prolonging Prophylactic Therapy in Healthy Subjects: First, Do No Harm. Journal of the American College of Cardiology, 75(25), 3184-3186. https://doi.org/10.1016/j.jacc.2020.05.008

González-Gay, M. A., Castañeda, S., \& Ancochea, J. (2021). Biologic Therapy in COVID-19. Archivos de Bronconeumologia, 57(1), 1-2. https://doi.org/10.1016/j.arbres.2020.06.007

Hamizi, K., Aouidane, S., \& Belaaloui, G. (2020). Etoposide-based therapy for severe forms of COVID-19. Medical Hypotheses, 142(April), 109826. https://doi.org/10.1016/j.mehy.2020.109826

Hariyanto, T. I., \& Kurniawan, A. (2020). Statin therapy did not improve the in-hospital outcome of coronavirus disease 2019 (COVID-19) infection. Diabetes and Metabolic Syndrome: Clinical Research and Reviews, 14(6), 1613-1615. https://doi.org/10.1016/j.dsx.2020.08.023

Homolak, J., \& Kodvanj, I. (2020). Widely available lysosome targeting agents should be considered a potential therapy for COVID-19. International Journal of Antimicrobial Agents, 56(2), 106044. https://doi.org/10.1016/j.ijantimicag.2020.106044

Huang, R., Zhu, C., Jian Wang, Xue, L., Li, C., Yan, X., Huang, S., Zhang, B., Zhu, L., Xu, T., Ming, F., Zhao, Y., Cheng, J., Shao, H., Zhao, X. an, Sang, D., Zhao, H., Guan, X., Chen, X., ... Wu, C. (2020). Corticosteroid therapy is associated with the delay of SARS-CoV-2 clearance in COVID-19 patients. European Journal of Pharmacology, 889(July), 173556. https://doi.org/10.1016/j.ejphar.2020.173556

Ip, A., Berry, D. A., Hansen, E., Goy, A. H., Pecora, A. L., Sinclaire, B. A., Bednarz, U., Marafelias, M., Berry, S. M., Berry, N. S., Mathura, S., Sawczuk, I. S., Biran, N., Go, R. C., Sperber, S., Piwoz, J. A., Balani, B., Cicogna, C., Sebti, R., ... Goldberg, S. L. (2020). Hydroxychloroquine and tocilizumab therapy in COVID-19 patients-An observational study. PLoS ONE, 15(8 August), 1-19. https://doi.org/10.1371/journal.pone.0237693

Izadi, M., Cegolon, L., Javanbakht, M., \& Sarafzadeh, A. (2020). Ozone therapy for the treatment of COVID-19 pneumonia: A scoping review. International Immunopharmacology, 92(January).

Jankowska, E. A., Sierpiński, R., Tkaczyszyn, M., Drozd, M., Szachniewicz, J., Duda-Sikuła, M., Knysz, B., Simon, K., Szenborn, L., \& Ponikowski, P. (2020). Chloroquine and hydroxychloroquine for the prevention and therapy of coronavirus disease 2019: new hopes and old cardiovascular concerns. Kardiologia Polska, 78(7-8), 811-817. https://doi.org/10.33963/KP.15511

Jha, A. K., Kumar, R., Goenka, M. K., \& Dayal, V. M. (2020). Emerging Treatment and Prevention Strategies against COVID-19: A Brief Update. Journal of Digestive Endoscopy, 11(01), 69-72. https://doi.org/10.1055/s-0040-1712547

Ji, F., Liu, W., Hao, D. A., Cheng, J., Tong, X. C., Hao, J. G., Wang, L. P., Li, C. Y., Dai, M. J., \& Yan, X. B. (2021). Use of convalescent plasma therapy in eight individuals with mild COVID-19. New Microbes and New Infections, 39, 100814. https://doi.org/10.1016/j.nmni.2020.100814

Kirsch, D. G. (2020). Radiation Therapy as a Treatment for COVID-19? International Journal of Radiation Oncology Biology Physics, 108(5), 1140-1142. https://doi.org/10.1016/j.ijrobp.2020.07.025

Klopfenstein, T., Zayet, S., Lohse, A., Balblanc, J. C., Badie, J., Royer, P. Y., Toko, L., Mezher, C., Kadiane-Oussou, N. J., Bossert, M., Bozgan, A. M., Charpentier, A., Roux, M. F., Contreras, R., Mazurier, I., Dussert, P., Gendrin, V., \& Conrozier, T. (2020). Tocilizumab therapy reduced intensive care unit admissions and/or mortality in COVID-19 patients. Medecine et Maladies Infectieuses, 50(5), 397-400. https://doi.org/10.1016/j.medmal.2020.05.001

Lana, R. M., Coelho, F. C., Da Costa Gomes, M. F., Cruz, O. G., Bastos, L. S., Villela, D. A. M., \& Codeço, C. T. (2020). The novel coronavirus (SARSCoV-2) emergency and the role of timely and effective national health surveillance. Cadernos de Saude Publica, 36(3). https://doi.org/10.1590/0102$311 \times 00019620$

Langhi, D. M., Santis, G. C. De, \& Bordin, J. O. (2020). COVID-19 convalescent plasma transfusion. Hematology, Transfusion and Cell Therapy, 42(2), 113115. https://doi.org/10.1016/j.htct.2020.04.003

Laurence, J., Mulvey, J. J., Seshadri, M., Racanelli, A., Harp, J., Schenck, E. J., Zappetti, D., Horn, E. M., \& Magro, C. M. (2020). Anti-complement C5 therapy with eculizumab in three cases of critical COVID-19. Clinical Immunology, 219(July), 108555. https://doi.org/10.1016/j.clim.2020.108555

Lawlor, M., Gupta, A., Ranard, L. S., Madhavan, M. V., Li, J., Eisenberger, A., Parikh, S. A., Sethi, S. S., \& Masoumi, A. (2021). Discordance in activated partial thromboplastin time and anti-factor Xa levels in COVID-19 patients on heparin therapy. Thrombosis Research, 198(June 2020), 79-82. https://doi.org/10.1016/j.thromres.2020.11.030 
Li, Q., Guan, X., Wu, P., Wang, X., Zhou, L., Tong, Y., Ren, R., Leung, K. S. M., Lau, E. H. Y., Wong, J. Y., Xing, X., Xiang, N., Wu, Y., Li, C., Chen, Q., Li, D., Liu, T., Zhao, J., Liu, M., ... Feng, Z. (2020). Early transmission dynamics in Wuhan, China, of novel coronavirus-infected pneumonia. New England Journal of Medicine, 382(13), 1199-1207. https://doi.org/10.1056/NEJMoa2001316

Li, S., Liu, C., Guo, F., Taleb, S. J., Tong, M., \& Shang, D. (2020). Traditional Chinese medicine a potential therapy for COVID-19. American Journal of Chinese Medicine, 48(6), 1263-1277. https://doi.org/10.1142/S0192415X20500627

Li, X., Yang, Y., Liu, L., Yang, X., Zhao, X., Li, Y., Ge, Y., Shi, Y., Lv, P., Zhang, J., Bai, T., Zhou, H., Luo, P., \& Huang, S. (2020). Effect of combination antiviral therapy on hematological profiles in 151 adults hospitalized with severe coronavirus disease 2019. Pharmacological Research, 160, 105036. https://doi.org/10.1016/j.phrs.2020.105036

Li, Y., Shi, K., Qi, F., Yu, Z., Chen, C., Pan, J., Wu, G., Chen, Y., Li, J., Chen, Y., Zhou, T., Li, X., \& Xia, J. (2021). Thalidomide combined with short-term low-dose glucocorticoid therapy for the treatment of severe COVID-19: A case-series study. International Journal of Infectious Diseases, 103, 507-513. https://doi.org/10.1016/j.ijid.2020.12.023

Lim, Z. J., Subramaniam, A., Reddy, M. P., Blecher, G., Kadam, U., Afroz, A., Billah, B., Ashwin, S., Kubicki, M., Bilotta, F., Curtis, J. R., \& Rubulotta, F. (2021). Case Fatality Rates for Patients with COVID-19 Requiring Invasive Mechanical Ventilation. American Journal of Respiratory and Critical Care Medicine, 203(1), 54-66. https://doi.org/10.1164/rccm.202006-2405OC

Liu, W., Zhu, H. L., \& Duan, Y. (2020). Virus-, host-, immune-based targets for COVID-19 therapy. Drug Discovery Today, 25(12), 2071-2073. https://doi.org/10.1016/j.drudis.2020.10.001

Liu, X., Long, C., Xiong, Q., Chen, C., Ma, J., Su, Y., \& Hong, K. (2020). Association of angiotensin-converting enzyme inhibitors and angiotensin II receptor blockers with risk of COVID-19, inflammation level, severity, and death in patients with COVID-19: A rapid systematic review and meta-analysis. Clinical Cardiology, July, 1-10. https://doi.org/10.1002/clc.23421

López-Alcalde, J., Yan, Y., Witt, C. M., \& Barth, J. (2020). The current State of Research about Chinese Herbal Medicines (CHM) for the Treatment of Coronavirus Disease 2019 (COVID-19): A Scoping Review. Journal of Alternative and Complementary Medicine, 26(7), 557-570. https://doi.org/10.1089/acm.2020.0189

Lovetrue, B. (2020). The AI-discovered etiology of COVID-19 and rationale of the irinotecan+ etoposide combination therapy for critically ill COVID-19 patients. Medical Hypotheses, 144(August), 110180. https://doi.org/10.1016/j.mehy.2020.110180

Mansourabadi, A. H., Sadeghalvad, M., Mohammadi-Motlagh, H. R., \& Rezaei, N. (2020). The immune system as a target for therapy of SARS-CoV-2: A systematic review of the current immunotherapies for COVID-19. Life Sciences, 258(June), 118185. https://doi.org/10.1016/j.lfs.2020.118185

Mattos-Silva, P., Felix, N. S., Silva, P. L., Robba, C., Battaglini, D., Pelosi, P., Rocco, P. R. M., \& Cruz, F. F. (2020). Pros and cons of corticosteroid therapy for COVID-19 patients. Respiratory Physiology and Neurobiology, 280(June), 103492. https://doi.org/10.1016/j.resp.2020.103492

Muchtaridi, M., Fauzi, M., Ikram, N. K. K., Gazzali, A. M., \& Wahab, H. A. (2020). Natural Flavonoids as Potential Angiotensin-Converting Enzyme 2 Inhibitors for Anti-SARS-CoV-2. Molecules, 25(17). https://doi.org/10.3390/molecules25173980

Nadaroglu, H. (2020). Antiviral drugs and plasma therapy used for COVID-19 treatment: a nationwide Turkish algorithm. Drug Metabolism Reviews, 52(4), 531-539. https://doi.org/10.1080/03602532.2020.1803907

Nagoba, B., Gavkare, A., Jamadar, N., Mumbre, S., \& Selkar, S. (2020). Positive aspects, negative aspects, and limitations of plasma therapy with special reference to COVID-19. Journal of Infection and Public Health, 13(12), 1818-1822. https://doi.org/10.1016/j.jiph.2020.08.011

Najar Nobari, N., Seirafianpour, F., Mashayekhi, F., \& Goodarzi, A. (2020). A systematic review on treatment-related mucocutaneous reactions in COVID-19 patients. Dermatologic Therapy, October 2020, 1-16. https://doi.org/10.1111/dth.14662

Nasonov, E., \& Samsonov, M. (2020). The role of Interleukin 6 inhibitors in therapy of severe COVID-19. Biomedicine and Pharmacotherapy, 131, 110698. https://doi.org/10.1016/j.biopha.2020.110698

Oladele, J. O., Ajayi, E. I., Oyeleke, O. M., Oladele, O. T., Olowookere, B. D., Adeniyi, B. M., Oyewole, O. I., \& Oladiji, A. T. (2020). A systematic review on COVID-19 pandemic with special emphasis on curative potentials of Nigeria-based medicinal plants. Heliyon, 6(9), e04897. https://doi.org/10.1016/j.heliyon.2020.e04897

OMS. (2020). Therapeutics and COVID-19. Living Guideline, November, 58.

Parasher, A. (2020). COVID-19: Current understanding of its pathophysiology, clinical presentation, and treatment. Postgraduate Medical Journal, 1(1), 1-9. https://doi.org/10.1136/postgradmedj-2020-138577

Patel, M., Gangemi, A., Marron, R., Chowdhury, J., Yousef, I., Zheng, M., Mills, N., Tragesser, L., Giurintano, J., Gupta, R., Gordon, M., Rali, P., D’Alonso, G., Fleece, D., Zhao, H., Patlakh, N., \& Criner, G. (2020). Retrospective analysis of high flow nasal therapy in COVID-19-related moderate-to-severe hypoxaemic respiratory failure. BMJ Open Respiratory Research, 7(1), 1-11. https://doi.org/10.1136/bmjresp-2020-000650

Poor, H. D., Ventetuolo, C. E., Tolbert, T., Chun, G., Serrao, G., Zeidman, A., Dangayach, N. S., Olin, J., Kohli-Seth, R., \& Powell, C. A. (2020). COVID-19 critical illness pathophysiology drove by diffuse pulmonary thrombi and pulmonary endothelial dysfunction responsive to thrombolysis. Clinical and Translational Medicine, 10(2), 3-7. https://doi.org/10.1002/ctm2.44

Procter, B. C., Ross, C., Pickard, V., Smith, E., Hanson, C., \& McCullough, P. A. (2021). Clinical outcomes after early ambulatory multidrug therapy for highrisk SARS-CoV-2 (COVID-19) infection. Reviews in Cardiovascular Medicine, 21(4), 611-614. https://doi.org/10.31083/J.RCM.2020.04.260

Rafiee, F., Keshavarz, P., Katal, S., Assadi, M., Nejati, S. F., Ebrahimian Sadabad, F., \& Gholamrezanezhad, A. (2021). Coronavirus Disease 2019 (COVID19) in Molecular Imaging: A Systematic Review of Incidental Detection of SARS-CoV-2 Pneumonia on PET Studies. Seminars in Nuclear Medicine, 51(2), 178-191. https://doi.org/10.1053/j.semnuclmed.2020.10.002 
Razmi, M., Hashemi, F., Gheytanchi, E., Dehghan Manshadi, M., Ghods, R., \& Madjd, Z. (2020). Immunomodulatory-based therapy as potentially promising treatment strategy against severe COVID-19 patients: A systematic review. International Immunopharmacology, 88(June), 106942. https://doi.org/10.1016/j.intimp.2020.106942

Rodríguez-Molinero, A., Pérez-López, C., Gálvez-Barrón, C., Miñarro, A., Rodríguez Gullello, E. A., Collado Pérez, I., Milà Ràfols, N., Mónaco, E. E., Hidalgo García, A., Añaños Carrasco, G., \& Chamero Pastilla, A. (2021). Association between high-dose steroid therapy, respiratory function, and time to discharge in patients with COVID-19: Cohort study. Medicina Clinica, 156(1), 7-12. https://doi.org/10.1016/j.medcli.2020.08.003

Russo, V., Di Maio, M., Attena, E., Silverio, A., Scudiero, F., Celentani, D., Lodigiani, C., \& Di Micco, P. (2020). Clinical impact of pre-admission antithrombotic therapy in hospitalized patients with COVID-19: A multicenter observational study. Pharmacological Research, 159, 104965. https://doi.org/10.1016/j.phrs.2020.104965

Saeedi Saravi, S. S., \& Beer, J. H. (2020). Apelin-potential therapy for COVID-19? Journal of Molecular and Cellular Cardiology, 145(June), 84-87. https://doi.org/10.1016/j.yjmcc.2020.06.007

Samaddar, A., Grover, M., \& Nag, V. L. (2020). Pathophysiology and Potential Therapeutic Candidates for COVID-19: A Poorly Understood Arena. Frontiers in Pharmacology, 11(585888), 1-23. https://doi.org/10.3389/fphar.2020.585888

Sauñe, P. M., Bryce-Alberti, M., Portmann-Baracco, A. S., \& Accinelli, R. A. (2020). Methylprednisolone pulse therapy: An alternative management of severe COVID-19. Respiratory Medicine Case Reports, 31(June), 12-14. https://doi.org/10.1016/j.rmcr.2020.101221

Seyedpour, S., Khodaei, B., Loghman, A. H., Seyedpour, N., Kisomi, M. F., Balibegloo, M., Nezamabadi, S. S., Gholami, B., Saghazadeh, A., \& Rezaei, N. (2020). Targeted therapy strategies against SARS-CoV-2 cell entry mechanisms: A systematic review of in vitro and in vivo studies. Journal of Cellular Physiology, June. https://doi.org/10.1002/jcp.30032

Seyhan, A. U., Doganay, F., Yilmaz, E., Topal, N. P., \& Ak, R. (2020). Investigation of QT prolongation with hydroxychloroquine and azithromycin for the treatment of COVID-19. Journal of the College of Physicians and Surgeons Pakistan, 30(10), S153-S157. https://doi.org/10.29271/jcpsp.2020.Supp2.S153

Shah, M., Captain, J., Vaidya, V., Kulkarni, A., Valsangkar, K., Nair, P. M. K., \& Ganu, G. (2021). Safety and efficacy of ozone therapy in mild to moderate COVID-19 patients: A phase 1/11 randomized control trial (SEOT study). International Immunopharmacology, 91(October 2020), 107301. https://doi.org/10.1016/j.intimp.2020.107301

Shereen, M. A., Khan, S., Kazmi, A., Bashir, N., \& Siddique, R. (2020). COVID-19 infection: Emergence, transmission, and characteristics of human coronaviruses. Journal of Advanced Research, 24, 91-98. https://doi.org/10.1016/j.jare.2020.03.005

Shi, C., Tingting, W., Li, J. P., Sullivan, M. A., Wang, C., Wang, H., Deng, B., \& Zhang, Y. (2021). Comprehensive Landscape of Heparin Therapy for COVID-19. Carbohydrate Polymers, 254(August), 117232. https://doi.org/10.1016/j.carbpol.2020.117232

Stremmel, C., Kellnar, A., Massberg, S., \& Kääb, S. (2020). Hydroxychloroquine in COVID-19 Therapy: Protection Versus Proarrhythmia. Journal of Cardiovascular Pharmacology and Therapeutics, 25(6), 497-502. https://doi.org/10.1177/1074248420935740

Sun, M., Xu, Y., He, H., Zhang, L., Wang, X., Qiu, Q., Sun, C., Guo, Y., Qiu, S., \& Ma, K. (2020). A potentially effective treatment for COVID-19: A systematic review and meta-analysis of convalescent plasma therapy in treating severe infectious disease. International Journal of Infectious Diseases, 98 , 334-346. https://doi.org/10.1016/j.ijid.2020.06.107.

Taha, M., Sharma, A., \& Soubani, A. (2020). Clinical deterioration during neutropenia recovery after G-CSF therapy inpatient with COVID-19. Respiratory Medicine Case Reports, 31, 101231. https://doi.org/10.1016/j.rmcr.2020.101231.

Takahashi, W., Yoneda, T., Koba, H., Ueda, T., Tsuji, N., Ogawa, H., \& Asakura, H. (2021). Potential mechanisms of nafamostat therapy for severe COVID19 pneumonia with disseminated intravascular coagulation. International Journal of Infectious Diseases, 102, 529-531. https://doi.org/10.1016/j.ijid.2020.10.093.

Tong, S., Su, Y., Yu, Y., Wu, C., Chen, J., Wang, S., \& Jiang, J. (2020). Ribavirin therapy for severe COVID-19: a retrospective cohort study. International Journal of Antimicrobial Agents, 56(3), 1-5. https://doi.org/10.1016/j.ijantimicag.2020.106114.

Trivedi, N., Verma, A., \& Kumar, D. (2020). Possible treatment and strategies for COVID-19: review and assessment. European Review for Medical and Pharmacological Sciences, 24(23), 12593-12608. https://doi.org/10.26355/eurrev_202012_24057.

Valizadeh, H., Abdolmohammadi-vahid, S., Danshina, S., Ziya Gencer, M., Ammari, A., Sadeghi, A., Roshangar, L., Aslani, S., Esmaeilzadeh, A., Ghaebi, M., Valizadeh, S., \& Ahmadi, M. (2020). Nano-curcumin therapy is a promising method in modulating inflammatory cytokines in COVID-19 patients. International Immunopharmacology, 89(June), 107088. https://doi.org/10.1016/j.intimp.2020.107088.

Veenstra, J., Buechler, C. R., Robinson, G., Chapman, S., Adelman, M., Tisack, A., Dimitrion, P., Todter, E., Kohen, L., \& Lim, H. W. (2020). Antecedent immunosuppressive therapy for immune-mediated inflammatory diseases in the setting of a COVID-19 outbreak. Journal of the American Academy of Dermatology, 83(6), 1696-1703. https://doi.org/10.1016/j.jaad.2020.07.089.

Vegivinti, C. T. R., Pederson, J. M., Saravu, K., Gupta, N., Evanson, K. W., Kamrowski, S., Schmidt, M., Barrett, A., Trent, H., Dibas, M., Reierson, N. L., Mikoff, N., Pisipati, S., Joseph, B. A., Selvan, P. T., Dmytriw, A. A., Pulakurthi, Y. S., Keesari, P. R., Sriram, V., ... Hassan, A. E. (2021). Efficacy of convalescent plasma therapy for COVID-19: A systematic review and meta-analysis. Journal of Clinical Apheresis, November 2020 , 1-13. https://doi.org/10.1002/jca.21881

Vianello, A., Arcaro, G., Molena, B., Turato, C., Sukthi, A., Guarnieri, G., Lugato, F., Senna, G., \& Navalesi, P. (2020). High-flow nasal cannula oxygen therapy to treat patients with hypoxemic acute respiratory failure consequent to SARS-CoV-2 infection. Thorax, 75(11), 998-1000. https://doi.org/10.1136/thoraxjnl-2020-214993

Wang, N., Zhan, Y., Zhu, L., Hou, Z., Liu, F., Song, P., Qiu, F., Wang, X., Zou, X., Wan, D., Qian, X., Wang, S., Guo, Y., Yu, H., Cui, M., Tong, G., Xu, Y., 
Research, Society and Development, v. 11, n. 2, e60011226307, 2022

(CC BY 4.0) | ISSN 2525-3409 | DOI: http://dx.doi.org/10.33448/rsd-v11i2.26307

Zheng, Z., Lu, Y., \& Hong, P. (2020). Retrospective Multicenter Cohort Study Shows Early Interferon Therapy Is Associated with Favorable Clinical Responses in COVID-19 Patients. Cell Host and Microbe, 28(3), 455-464.e2. https://doi.org/10.1016/j.chom.2020.07.005

Wiersinga, W. J., Rhodes, A., Cheng, A. C., Peacock, S. J., Prescott, H. C., \& Joost Wiersinga, W. (2020). Pathophysiology, Transmission, Diagnosis, and Treatment of Coronavirus Disease 2019 (COVID-19) AReview. JAMA, 324(8), 782-793. https://doi.org/10.1001/jama.2020.12839

Xu, P., Huang, J., Fan, Z., Huang, W., Qi, M., Lin, X., Song, W., \& Yi, L. (2020). Arbidol/IFN- $\alpha 2 b$ therapy for patients with coronavirus disease 2019: a retrospective multicenter cohort study. Microbes and Infection, 22(4-5), 200-205. https://doi.org/10.1016/j.micinf.2020.05.012

Yao, T. T., Qian, J. D., Zhu, W. Y., Wang, Y., \& Wang, G. Q. (2020). A systematic review of lopinavir therapy for SARS coronavirus and MERS coronavirus-A possible reference for coronavirus disease-19 treatment option. Journal of Medical Virology, 92(6), 556-563. https://doi.org/10.1002/jmv.25729

Yousefifard, M., Zali, A., Mohamed Ali, K., Madani Neishaboori, A., Zarghi, A., Hosseini, M., Safari, S., Ali, M. K., \& Neishaboori, M. A. (2020). Antiviral Therapy in Management ofCOVID-19: a Systematic Review on Current Evidence. Archives of Academic Emergency Medicine, 8(1), 45.

Zhao, M. (2020). Cytokine storm and immunomodulatory therapy in COVID-19: Role of chloroquine and anti-IL-6 monoclonal antibodies. International Journal of Antimicrobial Agents, 55(6), 105982. https://doi.org/10.1016/j.ijantimicag.2020.105982

Zhaori, G., Lu, L., Liu, C., \& Guo, Y. (2020). Progresses in clinical studies on antiviral therapies for COVID-19-Experience and lessons in the design of clinical trials. Pediatric Investigation, 4(4), 263-274. https://doi.org/10.1002/ped4.12227. 